\title{
El paisaje en animación suspendida y su valoración como dinamizador del desarrollo rural de la comuna de Maullín, Chile ${ }^{1}$
}

\author{
Ximena Galleguillos A.-Schübelin² y Carolina Ojeda Leal ${ }^{3}$
}

\begin{abstract}
RESUMEN
La percepción del paisaje por parte de los habitantes es expresión de identidad territorial, saberes comunitarios y la necesidad vital de preservación relativa a una conciencia de su fragilidad. Al ser un recurso natural con demanda creciente, el estudio de las preferencias territoriales éticas y estéticas, aporta conocimiento fundamental para una gestión ambiental sustentable. Utilizando la perspectiva etnometodológica, esta investigación analiza las percepciones y la valoración del paisaje en Maullín, Chile. El principal hallazgo, es el deseo de mantener el paisaje en animación suspendida, esto significa ralentizar la transformación del paisaje, y preservar su característica condición de tranquilidad. La rururbanización y la inminente transformación de estos territorios parece ir en una dirección opuesta y pone la identidad territorial y las aspiraciones de la comunidad en una tensión que requiere de una mediación. En este contexto, la consideración del enfoque de continuum urbano-rural donde el territorio urbano y rural constituye un solo gran sistema puede ser determinante para dinamizar el desarrollo territorial. ${ }^{4}$
\end{abstract}

Palabras clave: Paisaje en Animación Suspendida, Rururbanización, Gestión Ambiental Sustentable

\begin{abstract}
The perception and evaluation of the landscape by its inhabitants expressed their degrees of territorial identity and community knowledge regarding its fragility and vital need for preservation. Being a natural resource with growing demand, studies on ethical and aesthetic territorial preferences, provide fundamental knowledge for sustainable environmental management planning. Using an ethnomethodologically perspective, this research aims to analyze the perceptions and appreciation of the landscape in Maullin, Chile. The main finding is the desire to keep the landscape in suspended animation, this means slowing down the transformation of the landscape, and preserve its characteristic condition of tranquility. The rurbanization and the imminent transformation of these territories seem to go in an opposite direction and put the territorial identity and aspirations of the community in an effort that requires mediation. In this context, considering the approach of urban-rural continuum where urban and rural territory constitutes a single large system can be crucial to dinamize regional development.
\end{abstract}

Key words: Landscape in suspended animation, rurbanization, Sustainable Environmental Management

Este artículo incluye resultados de la investigación “Valoración del paisaje como potencial de Desarrollo Económico Sustentable. Comuna de Maullín - Chile" para optar al grado de Magister en Desarrollo Regional y Medio Ambiente, Universidad de Valparaíso (Chile). Las autoras agradecen a: Dr. Roberto Navarro, Prof. Karen Ojeda, Dra. Edilia Jaque y Dr. Cristian Echeverría por su colaboración. Además se agradece especialmente a Lorena Retamal encargada de DIDECO de la comuna de Maullín. Artículo recibido el 17 de noviembre de 2016 , aceptado el 28 de enero de 2016 y corregido el 28 de abril de 2016.

Universidad de Valparaíso (Chile). E-mail: galleguillos@geographie.uni-kiel.de

Universidad de Valparaíso (Chile). E-mail: carojedaleal@gmail.com 
El paisaje es inherente al hombre y está en una condición de reciprocidad vital. El territorio deviene paisaje a través de la interpretación cultural ${ }^{5}$. Tuan (2007) ilustra la diferencia de percepción del entorno entre un habitante local y un observador externo, en el sentido que mientras el primero tiene una percepción absolutamente basada en la experiencia (vivencia), el segundo tiene tiempo para asumir una posición distanciada que le permite racionalizar las experiencias intentando objetivarlas ${ }^{6}$ (Tuan, 2007). Cada paisaje puede analizarse para descubrir las fuerzas que lo originan, pero además nuestra relación con el paisaje es intuitiva y reflejo de una larga historia vivencial cultural. El distinto modo en que a cada cultura se le aparece su propio mundo es decir su relación con la tierra se denomina cosmofanía. Dicho concepto es una construcción del griego kosmos (mundo), y phanein, (aparecer) y con el que Berque (2009) valora y entiende las diferentes maneras en que se relaciona cada cultura con su entorno. La cosmofanía considera los relatos orales o escritos dedicados a la belleza de los lugares, la existencia de áreas de esparcimiento, una arquitectura planificada para disfrutar de hermosas vistas, pinturas que representen el entorno, una o varias palabras para referirse al paisaje y una reflexión explícita sobre el paisaje.

El Naturgeist (espíritu de la naturaleza) se ha manifestado al hombre desde el principio de los tiempos en una serie de lugares simbólicos, mágicos, llenos de imaginarios míticos que tienden a no variar mucho desde una cultura a otra: las cumbres, volcanes, islotes, fuentes de agua, se repiten por las más diversas localizaciones geográficas (Wunenburger, 2008) como si llevaran en sí el valor de su condición mítica. Esta inherencia del paisaje en el hombre se manifiesta en la cultura mapuche. "El aura del paisaje se acrecienta según la energía que la tierra emana en diferentes regiones o polos de tensión espiritual. Los mapuches de Chile llaman nehuén a ese espíritu que se posesiona de la tierra y los cuerpos y que acoge nuestro espíritu. El hombre que percibe el nehuén es porque ya posee el nehuén en su propio cuerpo"7 (Soublette, 2007: 25).

El paisaje aparece de acuerdo a la mirada de quien lo observa: este modo de ser del paisaje sugiere que no siempre exista para todos y que su aparecer se manifieste solo para algunos a quienes se les devela desde su sustrato geográfico. Los patrones culturales que ha expandido el consumo nos han conducido a una cosificación del paisaje. Sin embargo, el paisaje no es una cosa, no es un objeto grande ni un conjunto de objetos configurados por la naturaleza o transformados por la acción humana (Maderuelo, 2005: 17). No obstante, ese marco cultural que tamiza la mirada puede exceder esa dimensión estética para trasladarse hacia una dimensión ética que proyecta sobre el entorno la ideología de quien observa (Ritter, 1974). En este contexto, Castoriadis (2010) habla de un imaginario social que en su permanente mutación rige nuestro devenir cultural en la relación entre lo que imagina una sociedad y la institucionalización de esta visión social. Así, los imaginarios sociales que crean un propio mundo para la respectiva sociedad conforman la psique de los individuos y una representación del mundo, incluida la sociedad misma y su lugar en ese mundo (Castoriadis, 2010). Maderuelo (2005) reafirma la idea que el paisaje está

El origen del concepto de paisaje se localiza en las lenguas latinas junto a la palabra país, la tierra, el territorio geográfico, en otras palabras una base física desde donde solo por intermedio de la mirada del hombre es posible que aparezca (Roger, 2007; Maderuelo, 2005). Es el pagus el origen, pago se refiere a la tierra cultivada por la que en el Medioevo se pagaba impuestos. Significa distrito agrícola (pueblo, aldea). Quien habitaba esa tierra era el paganus (pagano) o el hombre de la tierra, de la comarca: el hombre rural (Maderuelo, 2005). Y en el lenguaje eclesiástico, recibió el nombre por la resistencia que la población rural ofreció a la cristianización (paganismo, paganizar). De esta raiz procede también el francés pays, territorio rural, y el castellano país; y paisaje en 1708, en francés paysage (Corominas, 1987: 433).

En el diálogo entre la antropóloga Dorothy Eggan y su informante hopi (Tuan, 2007: 90).

También desde la perspectiva del anclaje cultural-territorial se explicita esta idea de memoria del paisaje (en el interior de quien observa) en un colono alemán en Chile, en 1842: "No solo llevamos con nosotros un pasado sino también unos ambientes y unos paisajes" (Watsuji 2006: 40). 
mediado doblemente por la cultura en tanto que los hombres construyen la forma del paisaje, y en tanto además, que su ideología les permite a los hombres distinguirlo y valorarlo. El paisaje es una elaboración mental realizada a través de la cultura (Maderuelo, 2005: 17) .

La definición de paisaje, ha transitado de lo meramente estético a una combinación de elementos físicos, ecológicos y humanos, una superficie de terreno heterogénea, compuesta por un conjunto de ecosistemas en interacción que se repiten de forma similar en ella (Lowenthal 1962, González 1981a). Si consideramos al paisaje como el escenario de la actividad humana, cualquier acto repercute inmediatamente en los factores perceptuales. Entonces el paisaje percibido es una realidad física experimentable según el anclaje socio-cultural.

El paisaje como construcción socio-cultural (Laurie, 1975: 102) desconcierta por su complejidad, plasmada en las obras y relatos de innumerables viajeros, artistas (Reboratti, 2010: 9) poetas, arquitectos, ecólogos o investigadores (Ojeda, 2011a: 12), porque cada ser humano es un sujeto que aprecia, valora, percibe, modifica, deconstruye, reinventa, niega, olvida o evalúa constantemente un paisaje determinado en todo momento de su vida. Estas acciones van configurando un proceso de identificación que se pone en marcha todos los días, el que tiene su correlato en un impacto ambiental, a veces imperceptible en lo inmediato (Galleguillos, 2015; CLAES, 2010: 10).

Desde el momento en que se toma conciencia del paisaje, se marca una preferencia o gusto hacia elementos componentes del paisaje tales como la biodiversidad, geomorfología, arquitectura, infraestructura, equipamiento, colores, olores, iluminación, manufacturas, habitantes, etc. (Busquets y Cortina, 2009:110). Estas predilecciones hacia el paisaje de parte de los sujetos que lo habitan o visitan constituyen el objeto de análisis de los estudios de percepción y preferencias de paisajes, para establecer patrones y cuantificar preferencias. Esta aproximación es utilizada también en Evaluaciones de Impacto Ambiental (EIA) y en el desarrollo de intervenciones urbanísticas y proyectos arquitectónicos, para cualificar el paisaje que se desea intervenir, manejar o proteger (Muñoz-Pedreros et al, 2000:729; Kzam, 2008:2; Tévar, 1996:99). Los estudios con fines de observación ética y estética del paisaje aportan además en evidenciar el poder real que tiene el usuario o habitante en la construcción, destrucción y o abandono del territorio (García, 2015; Ballet, 2007: 21).

La valoración o desvalorización implica una intencionalidad que incide en las decisiones respecto del uso del suelo tanto a nivel individual como en la esfera pública y privada (Solano, 2013:78-79). En este contexto, en el análisis de preferencias de paisajes los actores (stakeholders) son personajes influyentes en el sentido común de la comunidad sobretodo por sus posiciones de poder, actividades profesionales, personales o aficiones: funcionarios de gobierno, actores de ONG's ambientalistas por ejemplo, o ligados a medios de comunicación, empresarios que desean invertir en el desarrollo comunitario, o en especulación en proyectos inmobiliarios (Ortegón et al., 2005: 55).

Desde que la Conferencia de Río en 1992 destacara las implicancias de la gobernanza participativa y la necesidad de tomar a los stakeholders como copartícipes en el manejo de recursos naturales, sus opiniones constituyen elementos de análisis fundamentales para las estrategias de desarrollo regional (Ballet et al., 2010: 54).

Varios autores y autoras (McCay \& Acheson, 1987:77; Agrawal \& Gibson, 1999:629-630; Conley \& Moote, 2003:37) destacan el hecho de que las instituciones locales y las comunidades que 
las apoyan, tienen un rol esencial en la eficiencia y manejo sustentable del paisaje natural como patrimonio natural, es decir el resguardo de los recursos naturales menos intervenidos por el ser humano. En este sentido, el imaginario social crecientemente asocia más los factores antrópicos que intervienen el territorio y menos, las propias reglas del sistema terrestre, respecto del manejo del medio ambiente natural (Hardin, 1968:1243). Muchas veces estos paisajes naturales se encuentran rodeados por comunidades que tienen la difícil decisión de conservarlos o intervenirlos, en la búsqueda de un mejor destino económico para sus habitantes (Plummer et al., 2014: 42) (Romero, 2014: 162).

El paisaje percibido y las preferencias sistematizadas desde un enfoque etnometodológico merecen especial consideración al momento de evaluar impactos ambientales negativos en un proyecto determinado. La planificación del paisaje incluye su preservación y conservación ecológica y la aplicación de un enfoque sistémico al conjunto rural-urbano. La percepción ambiental de las personas, debe ser fundamental en la gestión ambiental ${ }^{8}$.

Los estudios de valoración del paisaje son herramientas fundamentales de una gestión ambiental sustentable, que prioriza la identificación de preferencias territoriales éticas y estéticas, representativas de la propia identidad comunitaria de una determinada localidad ${ }^{9}$. Este constituye el principal propósito de esta investigación.

\section{Materiales y métodos}

Esta investigación sobre el paisaje y su valoración como dinamizador de desarrollo rural busca conocer las percepciones y la valoración que tienen los habitantes de la comuna de Maullín, Chile. Una comuna cuya vocación productiva ha sido por décadas silvoagropecuaria de carácter familiar, con explotación de plantaciones forestales nativas e introducidas, ganadería menor y comercio de sus subproductos -leche, carne, queso-, además de pesca artesanal y cultivo-extracción de mariscos y algas. Geográficamente, se ubica en la cuenca del río Maullín, además de ser su desembocadura natural y poseer humedales considerados como Reserva RAMSAR (Fernández et al., 2009:7).

El principal desafío está en la identificación de preferencias territoriales éticas y estéticas, representativas de la propia identidad comunitaria de la localidad de Maullín, contenido prioritario en la implementación de una gestión ambiental sustentable. Para ello se utilizó un diseño de investigación exploratorio de carácter cualitativo fundamentado en el enfoque etnometodológico

\footnotetext{
En Chile la evaluación de impacto ambiental de un proyecto está considerada por la Ley de Bases del Medio Ambiente, instruyéndose a quienes elaboren Estudios de Impacto Ambiental que consideren las áreas de singularidad paisajística en dichos estudios (CONAMA, 1993; CONAMA 1994). Por otro lado, la capacidad de acogida de un área turística está determinada por el equilibrio entre el número de visitantes y el grado de transformación que no implique efectos perjudiciales en los recursos (MOPT 1993) pero no incluye la percepción de paisaje y preferencias de sus habitantes; de este modo, es de vital importancia estimular, con este tipo de estudios, el poder evaluar con exactitud las preferencias y el potencial de acogida de un área determinada. Esto dimensionará las limitantes que existen, pero también determinará las potencialidades

9 La importancia del paisaje para una localidad es tal que los organismos, públicos y privados deberían obligarse por ley a poner en marcha acciones que permitan controlar el impacto ambiental que sus intervenciones territoriales ocasionan sobre el paisaje, especialmente cuando se trata de tomar decisiones frente a cambio de uso de suelos, propuestas de instalaciones industriales o facilidades públicas, proyectos de inversión en zonas de calidad y fragilidad paisajística.
} 
Cuadro $\mathrm{N}^{\circ} 1$

Caracterización de la selección de actores (stakeholders) entrevistados según criterio de compromiso e involucramiento en el desarrollo local

\begin{tabular}{|c|c|c|c|c|c|c|c|}
\hline \multirow{2}{*}{$\mathrm{N}^{\circ}$} & \multirow{2}{*}{ Nombre } & \multicolumn{3}{|c|}{ Tipo de organización } & \multirow{2}{*}{ Ubicación } & \multirow{2}{*}{ Impacto } & \multirow{2}{*}{$\begin{array}{l}\text { Importancia } \\
\text { estratégica }\end{array}$} \\
\hline & & Estatal & Privado & ONG & & & \\
\hline 1 & \begin{tabular}{|l} 
CECPAN (Centro \\
de Estudios y \\
Conservación del \\
Patrimonio Natural) \\
\end{tabular} & & & $\mathrm{x}$ & Pedro Montt 549 & Nacional & Alta \\
\hline 2 & $\begin{array}{l}\text { Comité Defensa Río } \\
\text { Maullín }\end{array}$ & & & $x$ & $S / n$ & Local & Alta \\
\hline 3 & \begin{tabular}{|l|} 
Dirección Desarrollo \\
Comunitario (DIDECO)
\end{tabular} & $\mathrm{x}$ & & & Edificio Consistorial & Local & Alta \\
\hline 4 & $\begin{array}{l}\text { Departamento de } \\
\text { Turismo Municipal }\end{array}$ & $x$ & & & Edificio Consistorial & Local & Alta \\
\hline 5 & Biblioteca Municipal & $x$ & & & Edificio Consistorial & Local & Media \\
\hline 6 & \begin{tabular}{|l|} 
Servicio Nacional de \\
Pesca (SERNAPESCA)
\end{tabular} & $\mathrm{X}$ & & & Edificio Consistorial & Local & Media \\
\hline 7 & Radio Maullín Fm & & $x$ & & Mac Iver s/n & Local & Alta \\
\hline 8 & \begin{tabular}{|l|} 
Radio Pangal Fm \\
\end{tabular} & & $x$ & & Subida Mac Iver s/n & Local & Alta \\
\hline 9 & $\begin{array}{l}\text { Mercado Municipal } \\
\text { (Cocina lafkenche) }\end{array}$ & & $x$ & & $\begin{array}{l}\text { Mercado Municipal } \\
\text { Local } 8\end{array}$ & Local & Alta \\
\hline 10 & \begin{tabular}{|l|} 
Director de Orquesta \\
Juvenil y Folklórica \\
Escuela Javier Abelardo \\
Núñez. \\
\end{tabular} & $x$ & & & Las Gardenias 500 & Local & Media \\
\hline 11 & $\begin{array}{l}\text { Representante Org. } \\
\text { Campesina "Las } \\
\text { orquídeas" de Puelpún. }\end{array}$ & & $x$ & & Puelpún s/n & Local & Alta \\
\hline 12 & Teatro Municipal Maullín & $x$ & & & O'Higgins 300 & Local & Baja \\
\hline 13 & $\begin{array}{l}\text { Oficina Juvenil } \\
\text { Municipalidad } \\
\end{array}$ & $x$ & & & Edificio Consistorial & Local & Media \\
\hline 14 & $\begin{array}{l}\text { Presidenta centro de } \\
\text { Padres Escuela Básica } \\
\text { Javier Abelardo Núñez. }\end{array}$ & & $x$ & & Maullín & Local & Baja \\
\hline 15 & \begin{tabular}{|l|} 
Artesana Taller laboral \\
"Delicias del estuario"
\end{tabular} & & $x$ & & Maullín & Local & Alta \\
\hline 16 & Taller laboral "Zafiro" & & $x$ & & Maullín & Local & Alta \\
\hline 17 & Fotógrafo & & $x$ & & Maullín & Local & Baja \\
\hline 18 & Artesana & & $x$ & & Maullín & Local & Baja \\
\hline 19 & $\begin{array}{l}\text { Unión Comunal de } \\
\text { Talleres Laborales }\end{array}$ & & & $x$ & Maullín & Local & Alta \\
\hline \multicolumn{2}{|c|}{ Total tipo de organismo } & 7 & 9 & 3 & \multicolumn{2}{|l|}{ Total importancia (Alta) } & 11 \\
\hline
\end{tabular}

Fuente: Elaboración propia. 
Figura $\mathrm{N}^{\circ} 1$

Etapas de la investigación

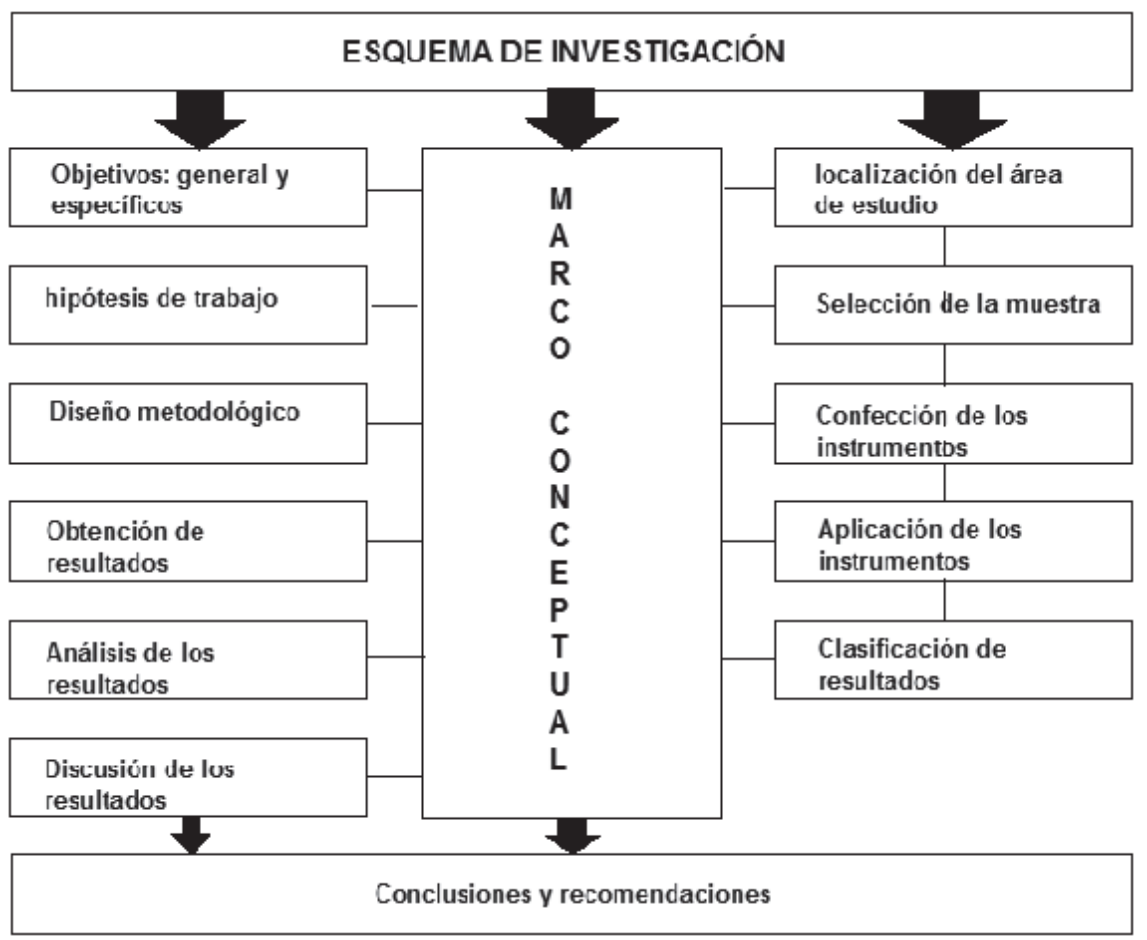

Fuente: Elaboración propia.

(Ibáñez, 1991: 2). La investigación se desarrolló en tres fases: indagación bibliográfica y marco teórico-metodológico, desarrollo empírico, percepción y valoración del paisaje a través de notas de terreno, y entrevistas estructuradas a actores (stakeholders) en la comuna de Maullín (Chile) los cuales fueron seleccionados en función del criterio cualitativo de involucramiento en el desarrollo local (dirigentes vecinales, periodistas, funcionarios del gobierno local y nacional, participantes en agrupaciones sociales $u$ ONG) ${ }^{10}$.

Como se puntualizó anteriormente, en el análisis de preferencias de paisajes los actores (stakeholders) son personajes influyentes en el sentido común de la comunidad (Ortegón et al., 2005: 55). Desde que la Conferencia de Río en 1992 destacara las implicancias de la gobernanza participativa y la necesidad de tomar a los actores (stakeholders) como copartícipes en el manejo de recursos naturales, sus opiniones constituyen elementos de análisis fundamentales para las estrategias de desarrollo regional (Ballet et al., 2010: 54). Las instituciones locales y las comunidades que las apoyan, tienen un rol esencial en la eficiencia y manejo sustentable del paisaje natural

\footnotetext{
El período de trabajo en terreno y entrevistas fue de un mes aproximadamente (Octubre 2013). La transcripción y análisis de las entrevistas realizadas en terreno otorga la perspectiva etnográfica (Cefaï, 2013:14). El análisis de discurso se hizo con el software ATLAS.TI 10 con su herramienta Wordcruncher, que facilita analizar las entrevistas en el aspecto discursivo textual (frases completas) y en la frecuencia en que un término es reiterado a lo largo del discurso.
} 
como patrimonio natural, es decir el resguardo de los recursos naturales menos intervenidos por el ser humano (Cuadro $N^{\circ} 1$ ).

\section{Percepción de la comuna y valoración del paisaje}

Los habitantes entrevistados reconocen y valoran su entorno natural y los elementos que componen este paisaje natural distinguiendo en él paisajes de mar (sailscapes) y paisajes acuáticos como el río Maullín (waterscapes), además de valorar las tradiciones, artesanías y patrimonio (Figura $\mathrm{N}^{\circ} 2$ ). No aparecen tipologías como las citadas por Busquets y Cortina (2009) tales como los paisajes sonoros (soundscapes), caminables (walkscapes), culturales (cultscapes), nubosos (cloudscapes), intervenidos con una perspectiva artística (artscapes).

Entrevistado 1: "Somos privilegiados por tener río y mar, es un privilegio que no todos tienen. Me encanta el agua".

Entrevistada 2: "A mí me encanta ver la lluvia. No me molesta".

Figura No 2

Frecuencia absoluta de aparición de términos durante las entrevistas

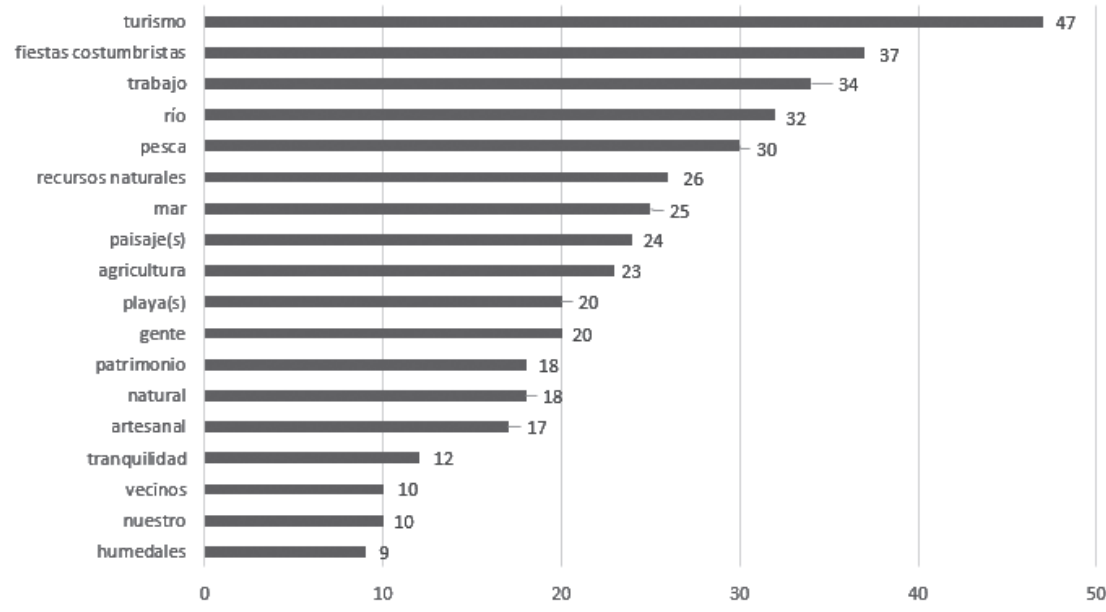

Fuente: Elaboración propia.

Los entrevistados reconocen además elementos bióticos éticos y estéticos, emocionales y económicos, que componen el paisaje natural, y el bienestar aparece asociado a la idea de estar rodeado de naturaleza, valoración que concuerda con observaciones de otros autores (Priego et al., 2014:54; Diener \& Seligman, 2004: 25):

Entrevistada 4: "Además el tener contacto con la naturaleza hace valorar la importancia de ésta y su preservación para las generaciones futuras. Algo totalmente opuesto a lo de grandes ciudades en donde las áreas verdes solo se reducen a los parques... iTener un árbol en casa es impagable!" 
Entrevistado 5: "A través de la música tratamos de mostrar el paisaje natural, mediante las canciones. (...) Tenemos mucha juventud que hace cultura en música, bandas jóvenes".

Los lugares conocidos y frecuentados mencionados por los entrevistados se cartografiaron con ArcGis 10 (Figura 3). Solá-Morales (2002) ha estudiado la vivencia del paisaje a través de la experiencia, y afirma que "la vida y el paisaje como totalidad están recogidas en espacios y lugares que solo nos son accesibles experimentándolos, viviéndolos" (Solá-Morales, 2002: 12). Para el caso de Maullín los lugares mencionados más frecuentados son:

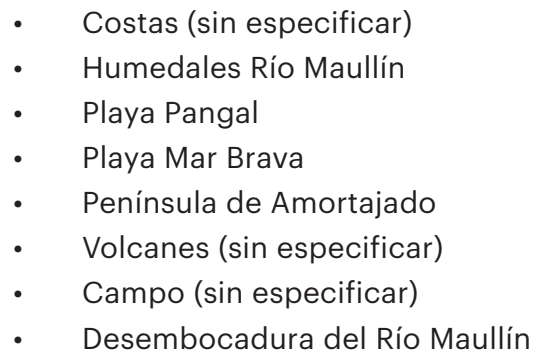

Los entrevistados perciben y mantienen en su memoria paisajes por los que transitan a diario y que ven periódicamente, a diferencia de otros paisajes que no conocen o que han visto solo en los medios de comunicación. Edney (1972), Nasar (1985) y Ojeda (2011b) en sus investigaciones argumentan que las experiencias vividas en un entorno conocido o la familiaridad que éste produce puede afectar la percepción del mismo. Esta información es valiosa en la elaboración de mapas perceptuales y valóricos del paisaje (Busquets y Cortina, 2009:100).

\section{Paisaje en animación suspendida}

El principal hallazgo, es la tendencia a querer mantener la condición de tranquilidad inalterable, impertérrita, que perciben y valoran los actores locales de Maullín, un paisaje en animación suspendida"1. Esta expresión de paisaje, definida por Smithson (1967), es una construcción temporal en la que pasado, presente y futuro no siguen necesariamente la secuencia temporal lineal a la que estamos acostumbrados, sino que más bien, giran en torno a los lugares de indeterminación, espacios en desuso y suspendidos en un tiempo indefinido (García, 2015). Estos llamados paisajes en animación suspendida se caracterizan por la lentitud o ausencia de transformaciones estructurales importantes en sus componentes, y en los imaginarios de sus habitantes evidencian la llamada añoranza rural o urbanismo romántico (Harvey, 2000: 37-39), es decir, vivir como en la ciudad manteniendo inalterado el paisaje. Las entrevistas evidencian recurrentemente un imaginario de paisaje en animación suspendida, o como lo retrata Smithson (1967) la idea de paisaje como construcción temporal no lineal: 
Figura $\mathrm{N}^{\circ} 3$

Lugares mencionados frecuentemente y con mayor valoración. Comuna de Maullín, Chile.

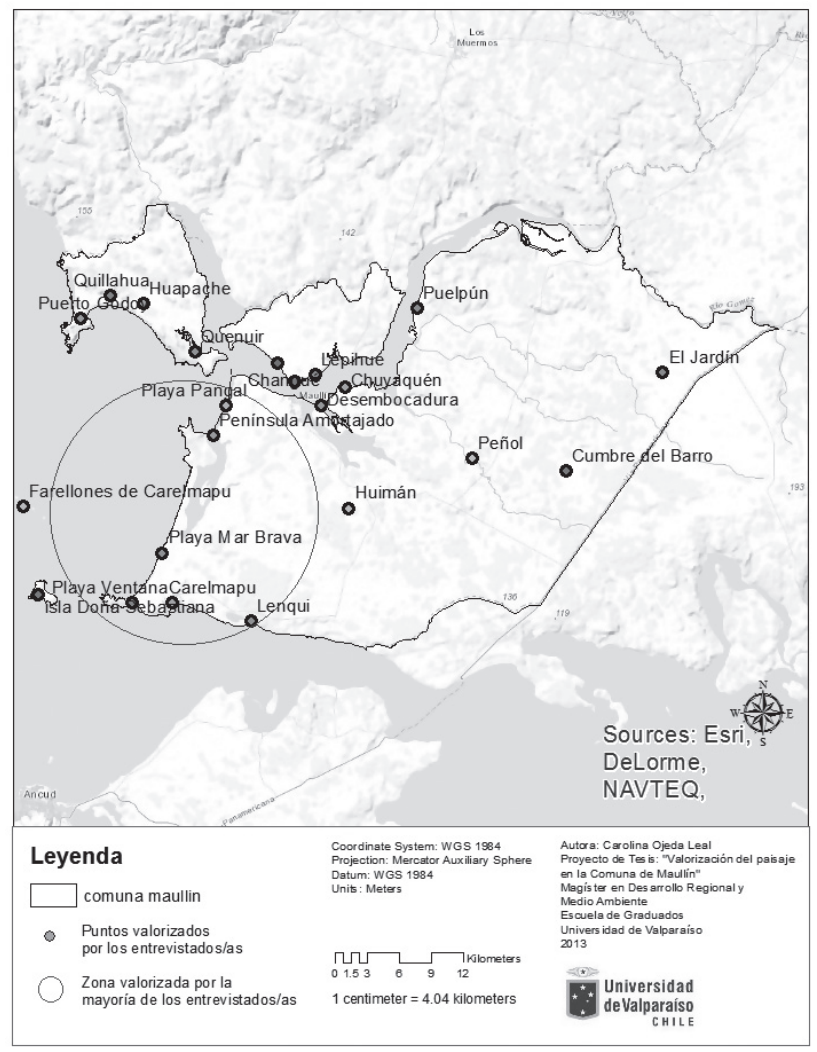

Fuente: Elaboración propia, 2014.

Entrevistada 6: "La comuna va a seguir igual".

Entrevistado 7: "La comuna va a seguir muy igual, como ahora".

Entrevistado 8: "Aún hoy siento que vivimos en el pasado, espero que esta situación no cambie".

Entrevistada 10: "La comuna va a seguir igual que hoy. Somos muy tranquilos acá. Porque la paz y tranquilidad son impagables. La tranquilidad del río es increíble, me permite componer mis canciones".

El paisaje se encuentra en un status quo inalterado y eso es un elemento identitario que les produce arraigo y motivación a permanecer en la comuna. No les atrae la modernidad que ellos traducen como el cemento urbano, o los shopping mall como elementos arquetípicos de consumo masivo, o el ajetreo en las calles. El shopping mall de Puerto Montt aparece citado como externalidad negativa (Figura № 4), reiterando que esta valoración negativa se produce sobre paisajes conocidos.

La tendencia en el discurso de los habitantes entrevistados en Maullín se puede asimilar al hecho que mientras las megatiendas gobiernan cada vez más el movimiento en las ciudades, la 
Figura $\mathrm{N}^{\circ} 4$

Vista del shopping mall Paseo Costanera 2014

Puerto Montt - Chile

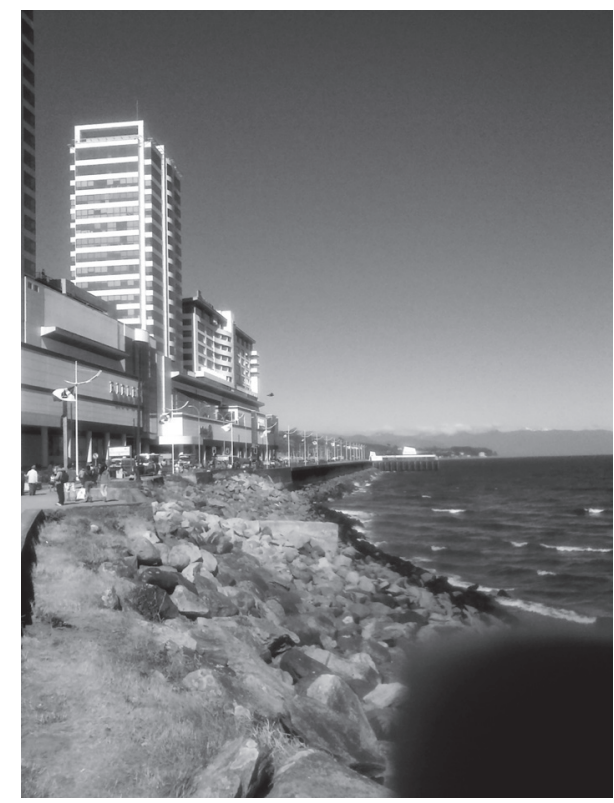

Fuente: Elaboración propia.

arquitectura y el urbanismo se presentan como meros coordinadores de este flujo, que va dejando a su paso insípidos espacios no arquitectónicos sin sentido que han llenado tantas ciudades genéricas $^{12}$ (Koolhaas et al., 2001; Ojeda, 2011b: 14; Costes, 2011: 95).

Los entrevistados en Maullín perciben la tranquilidad en la ausencia de ruido molesto, en la presencia de vegetación nativa e introducida con abundantes tonalidades verdes (White et al, 2013), en las viviendas de madera revestidas con zinc, asociadas a una arquitectura tradicional sureña ${ }^{13}$ (Figura 5), y a un hábitat rural con una intervención humana mínima dispersa en grandes extensiones de terreno (Mérida, 2011: 263).

En relación a la identidad territorial y la proyección futura los entrevistados se manifiestan a favor de mantener las costumbres:

Entrevistado 11: "Aún se mantiene la noción de comunidad, especialmente en las fiestas religiosas y el duelo, ambos rituales comunitarios. Sin la intervención que le quite su identidad, sin un mall ni cemento, especialmente utilizando materiales nobles como la madera".

\footnotetext{
"Se puede sostener que comprar es la última forma que queda de actividad pública. Las iglesias están imitando a los centros comerciales para atraer feligreses. Los arquitectos importantes desdeñan el mundo de las ventas al por menor, pero utilizan las configuraciones que ha dado lugar el comprar para diseñar museos y universidades. Las ciudades que pasan por dificultades son revitalizadas cuanto más se las plantea como centros comerciales" (Koolhaas, 2001:58).

13 Es un fenómeno muy similar a lo que la geografía crítica marxista denomina "producción de naturaleza", es decir, "la creación humana de un medio ambiente intervenido, por ejemplo a través de paisajes diseñados e incluso organismos genéticamente modificados" (Romero, 2014 162-163).
} 
Figura $\mathrm{N}^{\circ} 5$

Casa en Sector Rural de Maullín (Puelpún).

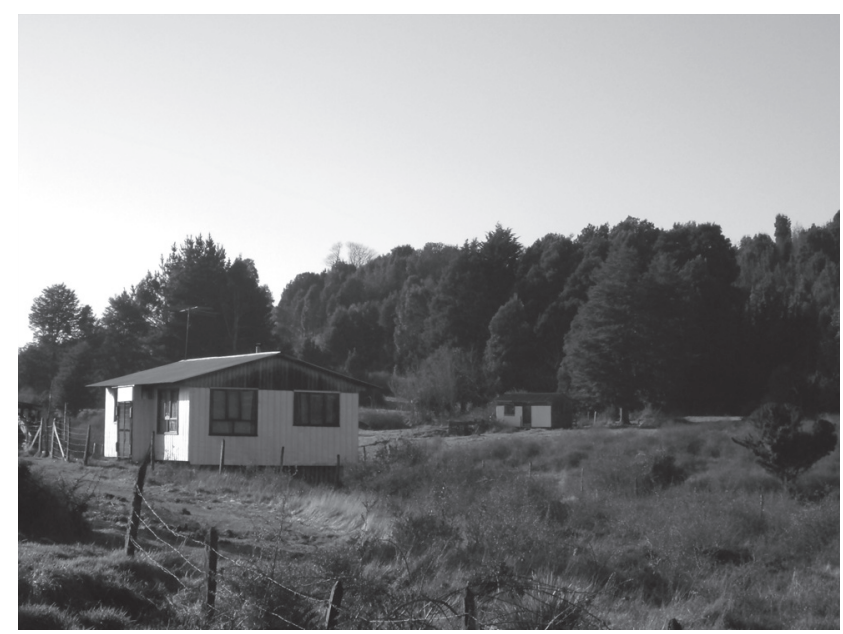

Fuente: Elaboración propia, 2014.

Entrevistada 12: "Me gusta la comuna, porque es tranquilo y tiene un paisaje bonito. No viviría en otra parte, menos en Puerto Montt... Ese mall es muy ruidoso y feo".

Entrevistado 13: "No me la imagino moderna, no creo que alguien haga un mall..."

\section{Tensión entre paisaje en animación suspendida y los desafíos que enfrenta la comuna en su desarrollo regional: el paisaje como recurso dinamizador}

Los conflictos ambientales por la intervención del territorio habitado por la comunidad, crean una tensión espacial (Lefebvre, 1972: 23). La existencia de conflictos ambientales derivados de la extracción de recursos naturales, forestales y agrícolas, así como del transporte, comunicaciones y turismo enfrentan el imaginario social de paisaje en animación suspendida al desafío de la transformación paisajística.

El modelo de rururbanización (paradojalmente anclado también en la añoranza rural y en la animación suspendida) y los modos de intervención territorial que se emplean actualmente, claramente van en la dirección opuesta, con claras consecuencias en el paisaje comunal observadas en terreno. Esta tensión se muestra en el discurso de los entrevistados, donde se expresan deseos de poseer ciertos servicios, facilidades y elementos propios de la vida urbana y que consideran como adelantos de la modernidad para la comuna y que han visto o conocido en las metrópolis cercanas -Puerto Montt o Puerto Varas-:

Entrevistada 15: "Me la imagino con alta tecnología, agua potable en los campos, caminos asfaltados". 
Entrevistada 16: "Yo me la imagino [a la comuna] con un par de industrias y turismo fluido, pavimentación en el acceso a la playa".

Estas aspiraciones, sin embargo, se abstraen del proceso de transformación que implica su implementación. La identidad comunitaria oscila entre una añoranza rural con calidad urbana, y la tensión que traen las respectivas transformaciones del paisaje. La comunidad de Maullín, que ha preferido mantener la tranquilidad y vivir en un paisaje en animación suspendida, transita hacia la rururbanización, y esto plantea la interrogante, si este tránsito implica también además de la identidad, la extinción del paisaje.

Como otras localidades de Chile, Maullín experimenta una rur-urbanización con un enfoque utilitarista de ciudad, el cual se basa en la idea en que las ciudades se desarrollan como centros de servicios mercantiles, funcionales a la actividad agrícola. Hace décadas Henri Lefebvre llamó la atención acerca de este proceso de utilitarización urbana y pérdida de identidad territorial: "la revolución urbana se está desarrollando ante nuestros ojos y está borrando la relación entre la ciudad y el campo fundando a ambos en lo urbano" (Lefebvre, 1972: 24).

Muy probablemente la rururbanización (que ya es un hecho) y sus habitantes van a requerir espacios para el abastecimiento, encuentro y diálogo ciudadano adecuados a esta nueva forma de habitar el territorio, con particularidades distintas a los espacios que existen en las metrópolis ${ }^{14}$. Al mismo tiempo, la cuestión es si la rururbanización será permeable a la identidad territorial como patrimonio material e inmaterial, manteniendo lugares memoriales, fiestas costumbristas, ferias y festividades religiosas, las que actualmente se desarrollan en la comuna de Maullín, de acuerdo al testimonio de los entrevistados.

La tensión que surge del enfrentamiento de las aspiraciones de un deseo de habitar un paisaje en animación suspendida, y la expansión de la rururbanización, es materia prioritaria de análisis. La frustración y desesperanza que produce la exclusión de las transformaciones en el territorio, incide directamente en su devenir. En este contexto, este fenómeno debe ser analizado desde un enfoque de urbanismo sustentable, como el del continuum Urbano-Rural cuya idea fuerza es que los sistemas urbanos y rurales constituyen un solo gran sistema (Dewey, 1960: 60-61) y donde las tensiones se resuelven en el marco de una gestión ambiental sustentable que considera la percepción y valoración del paisaje desde sus habitantes y su participación en la construcción social del territorio.

En la ciudad de Maullín se identifican zonas periurbanas sin uso establecido, y que son potenciales territorios de expansión. La expansión urbana que en forma predominante actúa con criterio económico, se hace presente en territorios que experimentan una fase temporal de inestabilidad económica, un declive de la agricultura y la falta de oportunidades de empleo rural (Allen, 2003:135) y en el caso de Maullín esta tendencia se muestra en el relato de los entrevistados sobre sus actividades productivas y ocupaciones (Cuadro No 2 ). 
Cuadro No 2

Rubros económicos descritos por los entrevistados/as

\begin{tabular}{|l|l|l|}
\hline $\begin{array}{l}|c| \\
\text { Pesca Artesanal y } \\
\text { Subproductos }\end{array}$ & \multicolumn{1}{|c|}{$\begin{array}{c}\text { Pequeño Comercio } \\
\text { (Importaciones) }\end{array}$} & \multicolumn{1}{c|}{$\begin{array}{c}\text { Pesca (Buceo) } \\
\text { y Subproductos }\end{array}$} \\
\hline $\begin{array}{l}\text { Pequeña Agricultura y } \\
\text { Subproductos }\end{array}$ & Servicios Públicos & $\begin{array}{l}\text { Pequeña Ganadería y } \\
\text { Subproductos }\end{array}$ \\
\hline Artesanías & $\begin{array}{l}\text { Turismo (Rural / Intereses } \\
\text { Especiales Como Avifauna- } \\
\text { Humedales) }\end{array}$ & Silvicultura y Subproductos \\
\hline
\end{tabular}

Fuente: Elaboración propia. Entrevistas procesadas mediante ATLAS.TI@, 2013.

Entre los efectos no deseados que junto a la rur-urbanización acelerada parecen ir en la dirección contraria a la valoración y percepción del paisaje, la expansión urbana sin regulación ni planificación estratégica y el cambio de uso de suelo desde el tradicional uso agrícola al forestal de corte monocultivo (pino y eucalipto) aparecen en los relatos de los entrevistados. En este sentido se puede argumentar que Maullín se encuentra en una fase de tránsito hacia una transformación del paisaje cuyas implicancias son la pérdida o desaparición de la identidad territorial. Es en este escenario donde la mediación ambiental y la inclusión de la participación de la comunidad en las decisiones de los destinos territoriales podría ser el primer paso de una gestión ambiental sustentable en el territorio que ponga en valor el paisaje en animación suspendida como dinamizador del desarrollo regional tanto para la comunidad, como para los inversionistas, y las instituciones gubernamentales encargadas del desarrollo regional (Casale et al., 2014).

\section{Conclusiones}

Los habitantes de la comunidad de Maullín reconocen como particularidad preponderante en el paisaje percibido y valorado el carácter natural, representado por los elementos agua del Río Maullín y la imagen del campo con sus bosques y granjas. Esta percepción y valoración se abstrae de la dimensión analítica compleja de las vulnerabilidades territoriales del paisaje que afectan a esta comuna.

La comunidad de Maullín, percibe y valora un desarrollo territorial que preserve un ritmo o de transformación del paisaje en animación suspendida, ralentizado. Esto quiere decir desarrollo y crecimiento económico sin perder su identidad y valores comunitarios. Los actores locales visualizan el desarrollo territorial orientado hacia el mantenimiento de una vida tranquila. Concretamente, el uso del suelo en grandes extensiones de terreno con habitat rural, y en la valoración positiva del sentimiento de tradicional calma sureña.

Esta percepción y valoración se enfrenta con la rururbanización generando una tensión en la identidad territorial. La animación suspendida es el mayor signo de arraigo territorial de las comunidades, y de rechazo inconsciente a una modernidad líquida, banal, de cemento, observada en la metrópolis. Esta tensión plantea la necesidad de reflexionar acerca de lo relativo de los procesos de desarrollo territorial. 
La valoración del paisaje como dinamizador de desarrollo regional, y la preservación del paisaje en animación suspendida, percibido como elemento identitario tradicional local, es valor agregado que se suma a los productos o servicios que ofrece la comunidad en Maullín. Las industrias creativas y el turismo pueden aportar a su mantención en ciclos sustentables. Un primer paso, es consolidar la valoración del paisaje como contenido prioritario en los planes de desarrollo local y estrategias comunales de desarrollo.

La percepción y valoración del paisaje presente proyectada al futuro muestra una ética y estética ambiental. La comunidad internaliza el paisaje cada vez más desde una conciencia ambiental que valoriza el paisaje natural como recurso natural vulnerable a su extinción y la necesidad de preservar sus ecosistemas. Esto explica la creciente resistencia ciudadana a la intervención de paisajes de alto valor natural cultural. La rururbanización y la inminente transformación de estos territorios parece ir en una dirección opuesta y pone la identidad territorial y las aspiraciones de la comunidad en una tensión que requiere de una mediación. La consideración del enfoque de continuum urbano-rural donde el territorio urbano y rural constituye un solo gran sistema y donde las tensiones se resuelven en el marco de una gestión ambiental sustentable que considera la percepción y valoración del paisaje desde sus habitantes y su participación en la construcción social del territorio, puede ser determinante para dinamizar el desarrollo territorial de la comuna de Maullín.

\section{Referencias bibliográficas}

AGRAWAL, A. \& GIBSON, C. Enchantment and disenchantment: The role of community in natural resource conservation. World Development, 1999, Vol. 27, N 4, p. 629-49.

ALLEN, A. Environmental planning and management of the peri-urban interface: perspectives on an emerging field. Environment and Urbanization, 2003, Vol.15, Nº1, p. 135-148.

BALLET, J. La gestion en commun des ressources naturelles, une perspective critique. Développement Durable et Territoires, 2007, № 29, p. 2-16.

BALLET, J.; KOFFI, J. \& BONIFACE, K. Co-Management of Natural Resources in Developing Countries : The Importance of Context. Économie Internationale, 2009, No 120, p. 53-76.

BERQUE, A. El pensamiento paisajero. Madrid. Biblioteca Nueva, 2009.

BUSQUETS, J. y CORTINA, A. Gestión del paisaje: Manual de Protección, Gestión y Ordenación del Paisaje. Barcelona. Ariel, 2009.

BORSDORF, A.; MOREIRA-MUÑOZ, A. y CASALE, J. (editores). Reservas de la Biósfera como Laboratorios para la Sustentabilidad: Paisajes de conservación y ordenamiento territorial. Santiago de Chile: Serie GEOlibros, Instituto de Geografía PUCCH - Academia de Ciencias Austríaca, 2014.

CASTORIADIS, C. Das imaginäre Element und die menschliche Schöpfung. Lich: Ausgewählte Schriften, Band 3 - Herausgegeben von Michael Halfbrodt und Harald Wolf. Übersetzt von Michael Halfbrodt. Auflage 1, Verlag Edition AV., 2010. 
CEFAÏ, D. ¿Qué es la etnografía? Segunda parte. Inscripciones, extensiones y recepciones del trabajo de campo. Persona y Sociedad, 2013, Vol. XXVII, N³, p. 11-32.

CLAES (CENTRO LATINOAMERICANO DE ECOLOGÍA SOCIAL). Tendencias en Ambiente y Desarrollo en América del Sur: Cambio Climático, biodiversidad y políticas ambientales (2009-2010). Montevideo: CLAES, 2010.

CONAMA (COMISION NACIONAL DEL MEDIO AMBIENTE). Pauta para la evaluación del impacto ambiental de proyectos de inversión. Instructivo Presidencial. Santiago de Chile: Comisión Nacional del Medio Ambiente, 1993.

CONAMA (COMISION NACIONAL DEL MEDIO AMBIENTE). Manual de evaluación de impactos ambientales: conceptos y antecedentes básicos. Santiago de Chile: Comisión Nacional del Medio Ambiente, Gobierno de Chile, 1994.

CONLEY, A. \& MOOTE, M. Evaluating collaborative natural resource management. Society and Natural Resources, 2003, Vol. 16, N5, p. 371-386.

COROMINAS, J. Diccionario Etimológico de la Lengua Castellana. Madrid: Gredos. 1987.

COSTES, L. Del "Derecho a la Ciudad" de Henri Lefebvre a la Universalidad de la Urbanización Moderna. Urban, 2011, № 502, p. 89-100.

DEWEY, R. The rural-urban continuum: Real, but relatively unimportant. American Journal of Sociology, 1960, Nº 1, p. 60-66.

DIENER, E. \& SELIGMAN, M. Beyond money: Toward an economy of well-being. Psychological Science in the Public Interest, 2004, Vol. 5, p. 1-31.

EDNEY, J. Place and Space: The Effects of Experience with a Physical Locale. Journal of Experimental Social Psychology, 1972, Nº 8, p. 50-62.

FERNÁNDEZ, L.; RAU, J., \& ARRIAGA, A. Calidad de la vegetación ribereña del Río Maullín $\left(41^{\circ} 28^{\prime}\right.$ '; $72^{\circ} 59^{\prime}$ W) utilizando el Índice QBR. Gayana Botánica, 2009, Vol. 66, № 2, p. 269-278.

GALLEGUILLOS, X. Valparaiso versus la dimension amigable del habitar. En: EGEA, C. \& SÁNCHEZ, D. (editores). Ciudades Amigables. Perspectivas, políticas, prácticas. Granada: Comares, 2015, p.129-150.

GARCÍA, C. Genealogía mínima del no-lugar. Esquimalenator, 2015. Disponible en Internet: http:// esquimalenator.wordpress.com/2012/09/08/genealogia-minima-del-no-lugar/

GONZÁLEZ F Ecología y paisaje. Madrid: H. Blume, 1981.

HARDIN, G. The tragedy of Commons. Science, 1968, N 162, p. 1243-48.

HARVEY, D. Megacities Lecture 4. Amsterdam: Twynstra Gudde Management Consultants. 2000. 
IBÁÑEZ, J. El Regreso del Sujeto. La investigación social de segundo orden. México: Amerinda, 1991.

KHZAM, E. La percepción ambiental como significación del paisaje: implicancias teóricas desde la relación del ser humano y el entorno. Revista Electrónica Ambiente Total, 2008, №1, p. 1-8.

KOOLHAAS, R.; CHUNG, C.; INABA, J.; LEONG, S.T. y CHA, T. Guía para comprar de la Escuela de Diseño de Harvard. New York: Taschen, 2001.

LAURIE, I. Aesthetic factors in visual evaluation. In: ZUBE, E.; BRUSH, R. \& FABOS, J. (editors). Landscape Assessment: Values, Perceptions and Resources. 1975. Stroudsburg: Dowden Hutchinson and Ross, p. 102-107.

LEFEBVRE, H. La Revolución Urbana. Madrid: Alianza Editorial, 1972.

LOWENTHAL, D. Not every prospect pleases. ¿What is our criterion for scenic beauty? Landscape, 1962, No 12, p. 19-23.

MADERUELO, J. Paisaje, génesis de un concepto. Madrid: Abada. 2005.

MCCAY, B. \& ACHESON, J. (editors). The Question of the Commons: The Culture and Ecology of Communal Resources. Tucson: University of Arizona Press, 1987.

MÉRIDA, M. La integración paisajística y sus fundamentos. Metodología de aplicación para construcciones dispersas en el espacio rural. Boletín de la Asociación de Geógrafos Españoles, 2011, $N^{\circ} 56$, p. 263-294.

MOPT (MINISTERIO DE OBRAS PUBLICAS Y TRANSPORTE). Guía metodológica para el estudio del medio físico y la planificación. Madrid: Ministerio de Obras Públicas y Transporte, Series Monográficas, 1993.

MUÑOZ-PEDREROS, A.; MONCADA-HERRERA, J. y LARRAÍN, A. Variación de la percepción del recurso paisaje en el sur de Chile. Revista Chilena de Historia Natural, 2000, Vol. 73, p. 729-738.

MUÑOZ-PEDREROS, A. La evaluación del paisaje: una herramienta de gestión ambiental. Revista Chilena de Historia Natural, 2004, Vol. 77, p. 139-156.

NASAR, J. Out of sight further from mind: Destination, visibility, and distance perception. Environment and Behavior, 1985, N 17, p. 50-62.

OJEDA LEAL, C. Análisis de fragilidad paisajística desde una perspectiva de género. Revista Digital para estudiantes de Geografía GeoGraphos, 2011a, Vol. 1, №4. Disponible en Internet: http://rua.ua.es/dspace/bitstream/10045/16631/1/no-4-2011-ojeda-leal.pdf

OJEDA LEAL, C. Estado del arte en las conceptualizaciones del paisaje y el paisaje urbano. Una revisión bibliográfica. Revista Digital para estudiantes de Geografía, GeoGraphos, 2011b, Vol. 4, Nº 
7. Disponible en Internet: http://web.ua.es/es/revista-geographos-giecryal/documentos/articulos/ no-7-2011-art-ojeda-leal.pdf?noCache 1305456946013

ORTEGÓN, E.; PACHECO, J. \& PRIETO, A. Metodología del Marco Lógico para la planificación, el seguimiento y la evaluación de proyectos y programas. Santiago de Chile: CEPAL-ILPES, 2005.

PLUMMER, R.; SITAS, N.; PROZESKY, H.; ESLER, K. \& REYERS, B. Opportunities and challenges for Mainstreaming Ecosystem Services in Development Planning: Perspectives from a Landscape Level. Landscape Ecology, 2014, Vol. 29, № 8, p. 1315-1331.

PRIEGO, C.; BREUSTE, J. \& RODRIGUEZ-MORCILLO, L. Naturaleza privada y calidad de vida. Influencia de la naturaleza doméstica en el bienestar de los propietarios de las casas con patio de la ciudad de Córdoba, España. Revista de Geografía Norte Grande, 2014, № 57, p. 54-64.

REBORATTI, C. La irresistible atracción del paisaje. Registros. Revista de Investigación Histórica, 2010, Vol. 7, No 7.

Disponible en Internet: http://faud.mdp.edu.ar/revistas/index.php/registros/ article/view/38

RITTER, J. Subjetividad. Barcelona: Editorial Alfa, 1974.

ROGER, A. Breve tratado del paisaje. Madrid/Buenos Aires: Biblioteca Nueva, 2007.

ROMERO TOLEDO, H. Ecología política y represas: elementos para el análisis del Proyecto Hidroaysén en la Patagonia chilena. Revista de Geografía Norte Grande, 2014, N57, p. 162-163.

SMITHSON, R. Recorrido por los monumentos de Passaic. New Jersey: Artforum, 1967.

SOLÁ-MORALES, I. Territorios. Barcelona: Gustavo Gili, 2002.

SOLANO, M. Imaginarios nacionales medioambientales en la espacialización comunitaria. Santiago de Chile: CLACSO - Serie Documentos de Trabajo Red de Posgrados, 2013. Disponible en Internet: http://bibliotecavirtual.clacso.org.ar/clacso/posgrados/20121227034502/OPMarianaSolanoUmanzor15.pdf

SOUBLETTE, G. La poética del acontecer. Santiago de Chile: Editorial Universitaria, 2007. TÉVAR, G. La cuenca visual en el análisis del paisaje. Serie Geográfica, 1996, Vol. 6, N¹, p. 99-113.

TUAN, Y. Topofilia. Madrid: Melusina, 2007.

WATSUJ, T. Antropología del paisaje. Salamanca: Editorial Sígueme, 2006.

WHITE, M.; ALCOCK, I.; WHEELER, B. \& DEPLEDGE, M. Would You Be Happier Living in a Greener Urban Area? A Fixed-Effects Analysis of Panel Data. Psychological Science, 2013, Vol. XX, No X, p. $1-9$.

WUNENBURGER, J. Antropología del imaginario. Buenos Aires: Ediciones del Sol, 2008. 
